\title{
FRAGILIDAD EN EL ADULTO MAYOR: DETECCIÓN, INTERVENCIÓN EN LA COMUNIDAD Y TOMA DE DECISIONES EN EL MANEJO DE ENFERMEDADES CRÓNICAS
}

\author{
Tania Tello-Rodríguez ${ }^{1,2, a}$, Luis Varela-Pinedo ${ }^{1,2, b}$
}

\begin{abstract}
RESUMEN
La fragilidad física constituye "un síndrome médico con múltiples causas y factores que contribuyen a su desarrollo, caracterizado por la disminución de la fuerza, resistencia y funciones fisiológicas reducidas, que aumentan la vulnerabilidad de un individuo en el desarrollo de la dependencia funcional y/o su muerte". Existen diferentes procesos fisiopatológicos multisistémicos involucrados en la patogénesis del síndrome de fragilidad, siendo uno de los más importantes la pérdida de masa muscular asociada al envejecimiento o sarcopenia. Diversos estudios indican que la fragilidad física se incrementa con la edad. El ejercicio físico constituye la mejor forma de intervención en la prevención y tratamiento de la fragilidad. En el adulto mayor con enfermedades crónicas identificar la fragilidad es muy importante para la toma de decisiones, individualizando el manejo y considerando sus valores y preferencias de cuidado.
\end{abstract}

Palabras clave: Anciano frágil; Anciano; intervención médica temprana; Enfermedad crónica (fuente: DeCS BIREME).

\section{FRAILTY IN OLDER ADULTS: DETECTION, COMMUNITY-BASED INTERVENTION, AND DECISION-MAKING IN THE MANAGEMENT OF CHRONIC ILLNESSES}

\begin{abstract}
Physical fragility is defined as "a medical syndrome with multiple causes and factors that contribute to its development and that is characterized by decreased strength and resistance as well as reduced physiological functioning, which increase an individual's vulnerability to functional dependence or death." Different multi-systemic pathophysiological processes are involved in the pathogenesis of frailty syndrome, one of the most important being the loss of muscle mass associated with aging or sarcopenia. Several studies have demonstrated that physical frailty increases with age. Physical exercise is the best form of intervention for preventing and treating frailty. In older adults with chronic illnesses, identifying frailty is very important for decision-making, individualizing management, and considering their values and care preferences.
\end{abstract}

Keywords: Frail elderly; Aged; Early medical intervention; Chronic disease (source: MeSH NLM).

\section{INTRODUCCIÓN}

La fragilidad física es definida, según el último consenso desarrollado por diferentes entidades destinadas al estudio del envejecimiento, como "un síndrome médico con múltiples causas y factores que contribuyen a su desarrollo, caracterizado por la disminución de la fuerza, resistencia y funciones fisiológicas reducidas que aumentan la vulnerabilidad de un individuo en el desarrollo de la dependencia funcional y/o su muerte" (1). Sin embargo, no hay un acuerdo sobre la mejor forma de medir u operacionalizar el diagnóstico de fragilidad. Dentro de las diversas escalas existentes para realizar el diagnóstico de fragilidad, las principales son el fenotipo de fragilidad de Linda Fried y el índice de fragilidad Rockwood y Mitnitsky. Linda Fried, en el año 2001, describió un fenotipo clínico de fragilidad que comprendía los siguientes criterios: pérdida intencional de peso de al menos el $5 \%$ en el último año, cansancio, baja actividad física, lenta velocidad de la marcha y debilidad muscular; considerando que un sujeto es frágil si cumple tres o más criterios, prefrágil si cumple uno o dos, y no frágil si no cumple ninguno de ellos (2). Este estudio demostró que algunos adultos mayores pueden ser frágiles y no tener discapacidad (compromiso en actividades de la vida diaria) ni comorbilidad (dos o

\footnotetext{
Instituto de Gerontología, Universidad Peruana Cayetano Heredia. Lima, Perú.

Hospital Nacional Cayetano Heredia. Lima, Perú.

Médico geriatra, magíster en Geriatría y Gerontología; ${ }^{\text {b }}$ médico internista-geriatra, doctor en Medicina

Recibido: 07/03/2016 Aprobado: 01/06/2016
}

Citar como: Tello-Rodríguez T, Varela-Pinedo L. Fragilidad en el adulto mayor: detección, intervención en la comunidad y toma de decisiones en el manejo de enfermedades crónicas. Rev Peru Med Exp Salud Publica. 2016;33(2)328-34.doi: 10.17843/rpmesp.2016.332.2207 
más enfermedades). Así pues, la fragilidad permite la evaluación de una dimensión que va más allá de lo que es medido apenas por el análisis de comorbilidades y capacidades funcionales ${ }^{(2-4)}$.

Rockwood y Mitnitsky elaboraron un constructo de fragilidad de acuerdo a la acumulación de déficits a diferentes niveles, incluyendo 70 ítems que corresponden a enfermedades, condiciones de salud, síndromes geriátricos o medidas de discapacidad ${ }^{(3,5)}$.

El envejecimiento es heterogéneo y es necesario determinar cuáles son los adultos mayores más débiles o vulnerables. En la práctica diaria nos preguntamos, por ejemplo, ¿por qué un paciente adulto mayor presenta complicaciones durante la hospitalización debido a una infección aguda o poscirugía, y por qué otros no? Para dar respuesta a esta interrogante presentamos diferentes escenarios clínicos:

Caso 1. El primer caso es un varón de 80 años con antecedente de hipertensión arterial, enfermedad pulmonar obstructiva leve e hipertrofia de próstata. Realizaba diariamente ejercicios, fue admitido para una cirugía electiva de hipertrofia prostática, tuvo una evolución adecuada, fue dado de alta a los 3 días y regresó a su estado basal funcional inmediatamente.

Caso 2. El segundo caso corresponde a un varón de 80 años con hipertensión arterial, enfermedad pulmonar obstructiva leve e hipertrofia de próstata, que habitualmente necesitaba ayuda para bañarse y vestirse. Durante el periodo posoperatorio, después de recibir un medicamento opiáceo para el dolor, presentó delirio y se quitó tanto la vía periférica como la sonda urinaria. En el manejo se le retiraron los opiáceos. Un familiar se quedó las $24 \mathrm{~h}$ del día, luego se le realizaron exámenes de laboratorio los cuales fueron normales. Se recuperó gradualmente y fue dado de alta 8 días después. El paciente regresó a su estado basal a las 2 semanas posteriores al alta.

Caso 3. Paciente de 81 años que vivía solo desde que su esposa murió hace 5 años, con historia de hipertensión arterial, enfermedad pulmonar obstructiva leve, y antecedente de cirugía de cadera izquierda y pérdida de peso $(8 \mathrm{~kg})$ en el último año. Usaba un bastón. Fue encontrado en el suelo por un vecino, se encontraba orientado, mencionó haberse caído desde una silla, no haber perdido la conciencia, con debilidad para levantarse. Fue evaluado en emergencia y hospitalizado, no se le encontraron fracturas ni alguna otra alteración en exámenes de laboratorio ni en estudios cardiovasculares por lo que fue referido a rehabilitación. Solo podía caminar con ayuda de un andador. Debido a que no podía cuidar de sí mismo, fue trasladado a una casa de reposo donde falleció cinco meses después.
Estas historias de casos ilustran el continuo de la fragilidad: un primer paciente, saludable o robusto, con poca vulnerabilidad a complicaciones de la cirugía; el segundo paciente, más vulnerable al factor estresante de la misma cirugía, presentó complicaciones posoperatorias, tomando mucho más tiempo su recuperación y, por último, el tercer paciente, era más claro que se trataba de un paciente frágil, falleció meses después.

La población está envejeciendo y, como se mencionó, en forma heterogénea, y esto tiene profundas implicaciones para la planificación y prestación de la atención sanitaria y social, convirtiéndose la fragilidad en una epidemia silenciosa que afecta a los adultos mayores ${ }^{(6)}$. Se conoce que hasta tres cuartas partes de las personas mayores de 85 años podrían no ser frágiles, lo que nos plantea interrogantes acerca de cómo se desarrolla la fragilidad y la forma en que podría ser prevenida y detectada de manera fiable para poder intervenir precozmente, por tratarse de una población que necesita una atención médica especial y que se constituirá en el grupo de usuarios frecuentes de los recursos sociosanitarios ${ }^{(7,8)}$.

\section{FISIOPATOLOGÍA}

Existen diferentes procesos fisiopatológicos multisistémicos en la patogénesis del síndrome de fragilidad, uno de los más importantes es la pérdida de masa muscular o sarcopenia asociada al envejecimiento. Fragilidad y sarcopenia se superponen: la mayoría de las personas de edad avanzada que son frágiles presentan sarcopenia y algunas personas de edad avanzada con sarcopenia también son frágiles ${ }^{(9)}$.

Existe, además, un estado de inflamación crónica y activación inmune, esto es probablemente un mecanismo subyacente clave que contribuye a la fragilidad directa e indirectamente. Diversos estudios han confirmado la asociación entre niveles elevados de interleuquina 6 y fragilidad. Otros factores etiológicos potenciales son los de causa genética, epigenéticos, metabólicos, neuroendocrinos, factores ambientales, de estilo de vida y la presencia de enfermedades agudas y crónicas ${ }^{(10-13)}$. (Figura 1)

\section{EPIDEMIOLOGÍA}

La prevalencia de fragilidad ha sido estimada en $6,9 \%$ en adultos mayores de la comunidad en los Estados Unidos; es mayor en mujeres y se incrementa con la edad, así, el 3,2\% pertenece al grupo entre los 65 a 70 años y el $23 \%$ a los mayores de 90 años ${ }^{(14)}$. En una 


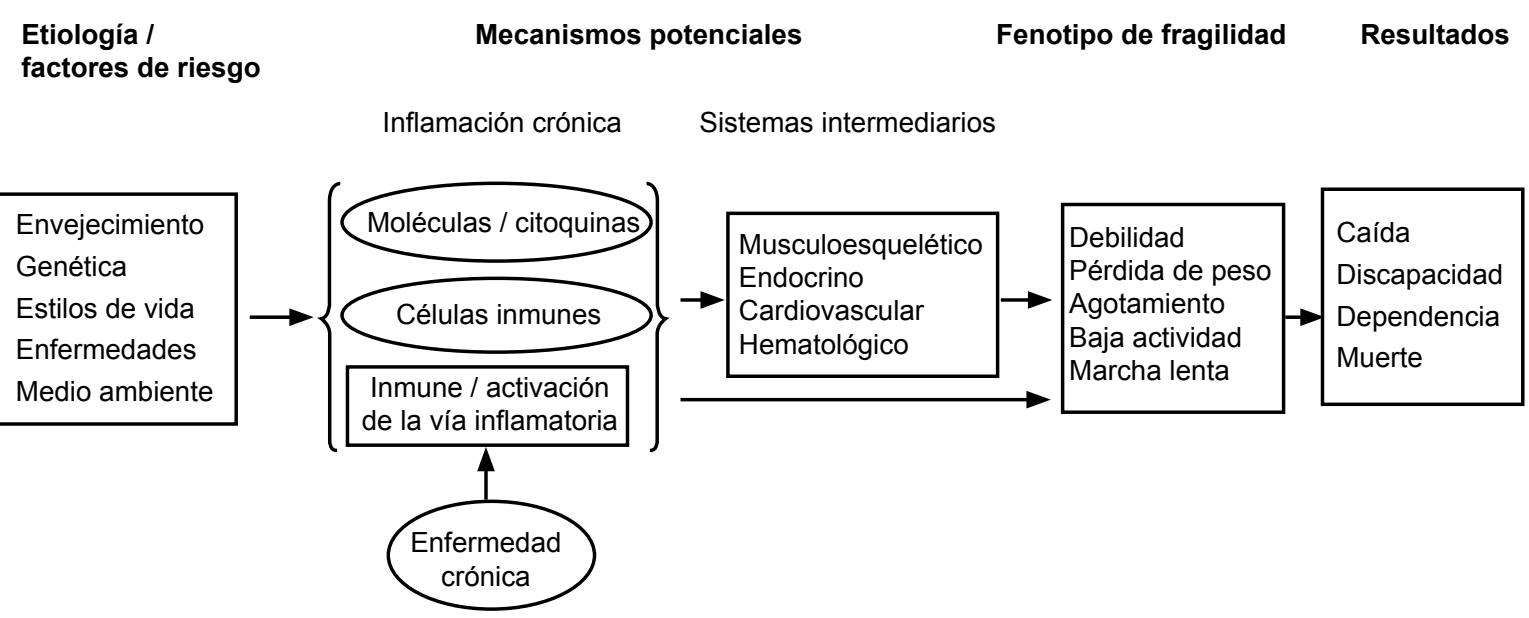

Adaptado de: Chen X, Mao G, Leng SX. Frailty syndrome: an overview Clin Interv Aging. 2014;9:433-41. doi: 10.2147/CIA S45300.

Figura 1. Patogénesis del síndrome de fragilidad: comprensión actual de los posibles mecanismos subyacentes y vías hipotéticas que conducirían a la fragilidad

revisión sistemática se encontró una prevalencia de 9,9\% para fragilidad física, incrementándose con la edad y en el sexo femenino ${ }^{(15)}$.

En el Cardiovascular Health Study (CHS) solamente el $9,7 \%$ de los adultos mayores con comorbilidad fueron frágiles, mientras que el $67,7 \%$ de adultos frágiles tenían alguna comorbilidad. El promedio de la cantidad de enfermedades crónicas en un adulto frágil fue 2,1 comparado con el 1,4 en no frágiles. Estos hallazgos sugieren que la fragilidad comparte algunos mecanismos con las enfermedades crónicas, una vez que las enfermedades han llegado a tener un severo o avanzado estado (2).

En una revisión sistemática sobre factores sociodemográficos y de salud asociados con la fragilidad en adultos mayores, se encontró que la edad, la raza negra y el sexo femenino mostraron una asociación positiva con la fragilidad, mientras que hubo una asociación inversa entre la fragilidad y la educación e ingresos económicos ${ }^{(16)}$.

En un estudio realizado en Lima Metropolitana en una muestra de 246 adultos mayores de la comunidad se encontró que, según los criterios de Fried, la frecuencia de fragilidad y prefragilidad fueron de $7,7 \mathrm{y}$ $64 \%$, respectivamente, y la frecuencia de comorbilidad y dependencia funcional fueron de 12,6 y $6,5 \%$, respectivamente, encontrándose, además, que el punto de corte de la velocidad de la marcha que determina fragilidad fue de $0,7 \mathrm{~m} / \mathrm{s}$. Asimismo, en un estudio realizado en adultos mayores de la comunidad del distrito de San Martín de Porres, se encontró que el 16,1\% presentaba fragilidad medida a través de la velocidad de la marcha $(<0,7 \mathrm{~m} / \mathrm{s})$, siendo más frecuente en mayores de 80 años, con un $40,3 \%{ }^{(17)}$.

\section{DETECCIÓN DE FRAGILIDAD}

Los dos modelos establecidos para la evaluación de fragilidad son el modelo de fenotipo del CHS y el modelo de déficit acumulado, correspondiente al Estudio Canadiense de Salud y Envejecimiento (Canadian Study of Health and Aging, CSHA). Ambos han sido validados en grandes estudios epidemiológicos, y son utilizados en estudios de investigación, pero no han sido desarrollados para su uso en la práctica diaria. El método usual de identificación de la fragilidad en la práctica clínica es la evaluación geriátrica integral, que requiere mucho tiempo, recursos y de un enfoque de especialista. Idealmente, la identificación de la fragilidad debiera darse en la comunidad, mediante pruebas simples y rápidas que puedan ser aplicadas por profesionales no especialistas ${ }^{(1,18)}$.

Se recomiendan algunos instrumentos de cribado desarrollados y validados para identificar personas frágiles, entre ellos tenemos la escala FRAIL (Clinical Frailty Scale) (Tabla 1), que de dar un resultado positivo obligan a una evaluación más profunda del paciente ${ }^{(1,19)}$.

En otros estudios se indica que la velocidad de la marcha (VM) es la prueba más adecuada para su uso en investigación y evaluación clínica; una lenta velocidad de la marcha fue identificada como marcador de complicaciones médicas relacionadas con la fragilidad (20). 
En una revisión sistemática sobre instrumentos simples para la identificación de fragilidad en adultos mayores de la comunidad, se encontró que la velocidad de la marcha, usando como un punto de corte $<0,8 \mathrm{~m} / \mathrm{s}$, tiene una alta sensibilidad $(0,99)$ y moderada especificidad $(0,64)$, mientras que un punto de corte de $<0,7 \mathrm{~m} / \mathrm{s}$ tiene una baja sensibilidad $(0,93)$, pero alta especificidad $(0,78)$; mientras que el instrumento "PRISMA 7 " y el test "Levántate y camina" demostraron alta sensibilidad y moderada especificidad para identificar fragilidad (18).

Por otro lado, un estudio de cohortes de adultos mayores que viven en la comunidad probó que la medición del estado de fragilidad usando el índice SOF (Study Osteoporotic Fractures) basada en tres componentes -pérdida de peso, dificultad para levantarse de una silla y pobre energía- es similar comparado con el CHS en la predicción de caídas, discapacidad, fractura y mortalidad ${ }^{(21)}$.

\section{INTERVENCIONES}

La reducción de la prevalencia y/o la severidad de fragilidad probablemente lleve a grandes beneficios para los adultos mayores, sus familias y la sociedad. Es más probable que los pacientes frágiles que recibieron una evaluación geriátrica integral y cuidados especiales retornen a casa con menor declive funcional, cognitivo y tengan menores tasas de mortalidad hospitalaria que aquellos que fueron admitidos a cuidados médicos generales ${ }^{(22)}$.

Existen numerosas causas potenciales de fragilidad física, muchas de ellas podrían ser intervenidas en el futuro. La calidad de la evidencia sobre las intervenciones para el tratamiento de la fragilidad es baja, y se necesitan más estudios con definiciones y mediciones de fragilidad estandarizados para determinar la eficacia de las intervenciones ${ }^{(23)}$

En la actualidad se describen cuatro posibles tratamientos que parecen tener alguna eficacia sobre la fragilidad: ejercicios aeróbicos y de resistencia, soporte

Tabla 1. Cuestionario FRAIL para detección de fragilidad en el adulto mayor

\begin{tabular}{l} 
Respuesta afirmativa: 1 a 2 = prefrágil; 3 o más = frágil \\
¿Está usted cansado? \\
¿Es incapaz de subir un piso de escaleras? \\
¿Es incapaz de caminar una manzana? \\
¿Tiene más de cinco enfermedades? \\
¿Ha perdido más del $5 \%$ de su peso en los últimos 6 meses? \\
\hline
\end{tabular}

FRAIL: Fatigue, Resistance, Aerobic, Illnesses, Loss of weight

Adaptado de: Morley JE, Vellas B, Van Kan GA, Anker SD, Bauer JM,

Bernabei R, et al. Frailty consensus: a call to action. J Am Med Dir Assoc. 2013;14(6):392-7. doi: 10.1016/j.jamda.2013.03.022. proteico y calórico, consumo de vitamina $\mathrm{D}$ y reducción de la polifarmacia ${ }^{(1,6)}$.

Yamada et al., en un programa de ejercicios aplicado a 610 personas frágiles de la comunidad, encontraron que el ejercicio (aeróbico, resistencia, flexibilidad y de equilibrio) en un programa estandarizado disminuyó la progresión de fragilidad y discapacidad (24). De la misma manera, en un metaanálisis se encontró que el grupo intervenido de personas frágiles con ejercicio durante $60 \mathrm{~min}$, dos veces por semana y con un seguimiento de al menos de ocho meses, mostró mejoras en diversos parámetros, como velocidad de la marcha, balance y mejora en las actividades de la vida diaria ${ }^{(25)}$.

En el estudio Lifestyle Interventions and Independence for Elders (LIFE) en el que los sujetos recibieron intervención con actividad física versus educación en envejecimiento exitoso, la prevalencia de fragilidad a los 12 meses fue significativamente baja en el grupo intervenido con actividad física (10\%), comparada con el grupo control que recibió educación en envejecimiento exitoso $(19,1 \%)^{(26)}$.

Los estudios clínicos aleatorizados de intervenciones nutricionales en personas frágiles siguen siendo escasos; uno de ellos es un estudio aleatorizado controlado que incluía 87 adultos mayores frágiles de la comunidad, con bajo estado socioeconómico, en los que recibieron suplementación nutricional por 12 semanas. Se observó que la funcionalidad física no cambió en el grupo control, pero sí en el grupo intervenido, en 5,9\%; la prueba de rendimiento físico declinó en $12,5 \%$ en el grupo control y permaneció estable en el grupo de intervención; la velocidad de la marcha disminuyó en ambos grupos, pero fue mayor en el grupo control $(11,3 \%)$, comparada con el grupo de intervención $(1,1 \%)^{(6,27)}$.

En otro estudio aleatorizado controlado se evaluó el impacto, a las 24 semanas de suplementación proteica, sobre la masa muscular, resistencia y rendimiento físico en adultos mayores frágiles, y se encontró que la masa muscular esquelética no cambió, la fuerza muscular del muslo se incrementó de $57 \pm 5 \mathrm{~kg}$ a $68 \pm 5 \mathrm{~kg}$ en el grupo intervenido, comparado con un incremento de $57,5 \mathrm{~kg}$ a 63,5 kg en el grupo placebo; el rendimiento físico mejoró significativamente en el grupo intervenido, pero no hubo cambios en el grupo placebo ${ }^{(28)}$.

Tieland et al. realizaron un estudio aleatorizado controlado en el que 62 adultos mayores frágiles participaron en un programa de ejercicios de resistencia dos veces por semana por 24 semanas, además, recibieron suplementos proteicos dos veces al día (30 gramos) o placebo, y se observó que la masa magra corporal se incrementó de $47,2 \mathrm{~kg}$ a $48,5 \mathrm{~kg}$ en el grupo 
intervenido, no hubo cambios en el grupo placebo; la fuerza muscular y el rendimiento físico mejoraron significativamente en ambos grupos ${ }^{(29)}$.

Existe evidencia de que la suplementación de vitamina $D$ en adultos mayores con deficiencia de $25(\mathrm{OH})$ vitamina $\mathrm{D}$ puede reducir caídas, fracturas de cadera, y esto podría también mejorar la función muscular. No existen grandes estudios clínicos que muestren que la fragilidad puede ser prevenida o tratada solamente con vitamina $D^{(1,30)}$.

La polifarmacia se reconoce como un posible factor importante que contribuya a la patogénesis de fragilidad, por lo que la reducción de medicamentos inapropiados puede disminuir claramente costos y efectos secundarios de la medicación en poblaciones frágiles ${ }^{(1)}$.

En una revisión sistemática sobre intervenciones para prevenir discapacidad en adultos mayores frágiles de la comunidad, no se encontró evidencia del efecto de la intervención nutricional sobre medidas de discapacidad, y de las ocho intervenciones de ejercicio físico, solamente en tres se reportaron resultados positivos para prevenir discapacidad (31).

Existe un concepto emergente en que la fragilidad puede ser considerada como un marcador de edad biológica, tal como se observa en estudios donde el estrés oxidativo estuvo asociado con fragilidad, pero no con edad cronológica, probablemente debido a que la fragilidad física es un mejor indicador de edad biológica, por lo que prevenir o revertir el desarrollo de la fragilidad física por medio de la práctica de ejercicio físico y hábitos de vida saludables sería equivalente a mantenerse joven biológicamente ${ }^{(32,33)}$.

\section{TOMA DE DECISIONES}

En la actualidad, los profesionales de la salud tenemos que atender a una población de edad cada vez más avanzada y con mayor comorbilidad y/o discapacidad, por lo que es necesario el conocimiento sobre fragilidad y su aplicación en distintas enfermedades médicas crónicas para poder individualizar su manejo ${ }^{(34)}$.

La fragilidad puede ser un método pronóstico, es decir, ayuda para la toma de decisiones. La adopción de cuidados invasivos, conservadores o paliativos no solo dependen del pronóstico de los pacientes, sino, sobre todo, de sus valores y preferencias de cuidado ${ }^{(35)}$.

\section{FRAGILIDAD Y ENFERMEDADES CARDIOVASCULARES}

En diferentes estudios se ha visto que la presencia de fragilidad en un adulto mayor con enfermedades cardiovasculares le confiere un aumento de dos veces en la mortalidad, efecto que persiste incluso después de hacer los ajustes por edad y comorbilidades. El impacto de la fragilidad se ha demostrado en enfermedades cardiovasculares estables, enfermedades cardiovasculares subclínicas, insuficiencia cardiaca, síndromes coronarios, cirugía cardiaca y en el reemplazo de la válvula aórtica transcatéter (TAVI) ${ }^{(36)}$. Ekerstad et al. realizaron un estudio prospectivo de 307 pacientes con infarto agudo de miocardio sin elevación del segmento ST; en el análisis multivariado la fragilidad mostró asociación con los eventos adversos cardiacos mayores $(\mathrm{OR}=2,2)$ y con la mortalidad ajustada al cabo de un mes $(\mathrm{OR}=4,7)^{(37)}$.

En un estudio en pacientes frágiles sometidos a reemplazo de la TAVI, la mortalidad inmediatamente después del procedimiento aumentó significativamente en los pacientes frágiles versus los no frágiles ( 5,5 vs $1,3 \%$; $p=0,04)$; la mortalidad al mes del reemplazo de la TAVI fue de $17 \%$ vs $5,8 \%(p=0,002)$ y la mortalidad a los 18 meses fue de $56 \%$ versus el $24 \%$ de aquellos $\sin$ fragilidad $(p<0,001)^{(38)}$.

\section{FRAGILIDAD Y DIABETES}

Los adultos mayores con diabetes es una población heterogénea con respecto a las comorbilidades, funcionalidad, nivel cognitivo, fragilidad, estado social y expectativa de vida, y es necesario conocerlas para el establecimiento de metas y tratamientos individualizados. Por ello, en los últimos años se ha incorporado en las guías clínicas de diabetes objetivos de tratamiento de acuerdo al estado de salud del adulto mayor, con el fin de prevenir el riesgo de hipoglicemia, mejorar la calidad de vida, mantener el estado funcional y evitar admisiones hospitalarias de complicaciones relacionadas con la diabetes ${ }^{(39)}$.

\section{FRAGILIDAD Y ONCOLOGÍA}

El cáncer afecta de manera desproporcionada a las personas mayores. Más de un tercio de los cánceres diagnosticados son en mayores de 70 años. Los pacientes adultos mayores con cáncer a menudo son tratados insuficientemente, están subrepresentados en los ensayos clínicos y tienen peores resultados que los individuos más jóvenes. La edad cronológica por sí sola es un pobre predictor de la tolerancia al tratamiento del cáncer, y la heterogeneidad de las personas mayores con cáncer requiere de un cuidadoso enfoque, tomando en cuenta la fragilidad del individuo. Actualmente, una persona de 65 años tiene una expectativa de vida en promedio de 18,5 años y una persona de 85 años, 6,4 años más. Esto representa un considerable número de años al final de la vida entrelazándose cada vez más con el cáncer. El tratamiento del cáncer debe ser enfocado en mantener o fortalecer la calidad de esos años ${ }^{(40)}$. 
En una revisión sistemática se encontró que la prevalencia de fragilidad y prefragilidad en pacientes con cáncer fue de $42 \%(6-86 \%)$ y $43 \%$ (13-79\%), respectivamente; un $32 \%$ fue clasificados como robusto; la fragilidad fue asociada independientemente con incremento de toda causa de mortalidad ajustada a los 5 años (HR: $1,87)$. Se encontró evidencia de incremento de riesgo de mortalidad posoperatoria a los 30 días para frágiles y prefrágiles. Las complicaciones postratamiento fueron más frecuentes en los frágiles, incluyendo la intolerancia al tratamiento de cáncer $(\mathrm{OR}=4,86)$ y complicaciones posoperatorias a los 30 días $(\mathrm{HR}=3,19)^{(41)}$.

\section{CONCLUSIONES}

La fragilidad está asociada a eventos adversos en los adultos mayores, por lo que es necesario que todos los profesionales de salud, especialmente los de atención primaria, identifiquen este grupo de adultos mayores para poder intervenir. El ejercicio físico constituye la mejor forma de intervención en la prevención y tratamiento de la fragilidad. Es necesario realizar estudios de investigación para validar instrumentos de cribado que sean aplicables en nuestro país. En el adulto mayor con enfermedades crónicas identificar la fragilidad es muy importante para la toma de decisiones, individualizando el manejo y de acuerdo con sus valores y preferencias de cuidado.

Contribuciones de autoría: LVP y TTR han participado conjuntamente en la concepción del artículo, su redacción y aprobación de la versión final.

Fuentes de financiamiento: autofinanciado.

Conflictos de interés: los autores declaran no tener conflictos de interés.

\section{REFERENCIAS BIBLIOGRÁFICAS}

1. Morley JE, Vellas B, Van Kan GA, Anker SD, Bauer JM, Bernabei R, et al. Frailty consensus: a call to action. J Am Med Dir Assoc. 2013;14(6):392-7. doi: 10.1016/j.jamda.2013.03.022.

2. Fried LP, Tangen CM, Walston J, Newman AB, Hirsch C, Gottdiener J, et al. Frailty in older adults: evidence for a phenotype. J Gerontol A Biol Sci Med Sci. 2001;56(3):M146-156. doi: 10.1093/gerona/56.3.M146

3. Abizanda P,Atienzar P, Garcia I. Fragilidad y sarcopenia. En: Abizanda P, Romero L, Luengo C, Sánchez $\mathrm{P}$, Jordan TJ. Medicina geriátrica. Barcelona: Elsevier; 2012. pp. 165-74.

4. Fried LP, Ferrucci L, Darer J, Williamson JD, Anderson G. Untangling the concepts of disability, frailty, and comorbidity: implications for improved targeting and care. J Gerontol A Biol Sci Med Sci. 2004;59(3):255-63. doi: 10.1093/ gerona/59.3.M255

5. Rockwood K, Mitnitski A, Song X, Steen B, Skoog I. Long-term risks of death and institutionalization of elderly people in relation to deficit accumulation at age 70. J Am Geriatr Soc. 2006; 54(6):975-9. doi: 10.1111/j.1532-5415.2006.00738.x.

6. Michel JP, Cruz-Jentoft AJ, Cederholm T. Frailty, Exercise and Nutrition. Clin Geriatr Med. 2015;31(3):375-87. doi: 10.1016/j.cger.2015.04.006.

7. Clegg A, Young J, lliffe S, Rikkert MO, Rockwood K. Frailty in elderly people.
Lancet. 2013;381(9868):752-62. doi: 10.1016/S0140-6736(12)62167-9.

8. Buckinx F, Rolland Y, Reginster JY, Ricour C, Petermans J, Bruyere O. Burden of frailty in the elderly population: perspectives for a public health challenge. Arch Public Health. 2015;73(1):19. doi: 10.1186/s13690015-0068-x.

9. Bauer JM, Sieber CC. Sarcopenia and frailty: a clinician's controversial point of view. Exp Gerontol. 2008;43(7):6748. doi: 10.1016/j.exger.2008.03.007.

10. Chen X, Mao G, Leng SX. Frailty syndrome: an overview. Clin Interv Aging. 2014;9:433-41. doi: 10.2147/ CIA.S45300.

11. Leng S, Fried LP. Inflammatory markers and Frailty. In: Fulop T, Franceschi C, Hirokawa K, Pawelec G, editors. Handbook on Inmunosenescence: Basic Understanding and Clinical Applications. New York: Springer; 2009:1293-303.

12. Yao X, Li H, Leng SX. Inflammation and immune system alterations in frailty. Clin Geriatr Med. 2011;27(1):79-87. doi: 10.1016/j.cger.2010.08.002.

13. Li HF, Manwani B, Leng S. Frailty, inflammation, and immunity. Aging Dis. 2011;2(6):466-73.

14. Weiss CO. Frailty and Chronic Diseases in Older Adults. Clin Geriatr Med. 2011;27(1):39-52. doi: 10.1016/j. cger.2010.08.003.
15. Collard RM, Boter H, Schoevers RA, Oude Voshaar RC. Prevalence of frailty in community-dwelling older persons: a systematic review. J Am Geriatr Soc. 2012;60(8):1487-92. doi: 10.1111/j.1532-5415.2012.04054.x.

16. Mello Ade C, Engstrom EM, Alves LC. Health-related and socio-demographic factors associated with frailty in the elderly: a systematic literature review. Cad Saude Publica. 2014;30(6):1143-68.

17. Varela Pinedo L, Ortiz Saavedra PJ, Chávez Jimeno H. Velocidad de la marcha como indicador de fragilidad en adultos mayores de la comunidad en Lima, Perú. Rev Esp Geriat Gerontol. 2010;45(1):22-5. doi: 10.1016/j. regg.2009.07.011.

18. Clegg A, Rogers L, Young J. Diagnostic test accuracy of simple instruments for identifying frailty in communitydwelling older people: a systematic review. Age Ageing. 2015;44(1):14852. doi: 10.1093/ageing/afu157.

19. Rockwood K, Song X, MacKnight C, Bergman H, Hogan DB, McDowell I, et al. A global clinical measure of fitness and frailty in elderly people. CMAJ. 2005;173(5):489-95.

20. Pamoukdjian F, Paillaud E, Zelek L, Laurent M, Lévy V, Landre $\mathrm{T}$, et al. Measurement of gait speed in older adults to identify complications associated with frailty: A systematic review. J Geriatric Oncol. 2015;6(6):484-96. doi: $10.1016 / j$. jgo.2015.08.006. 
21. Ensrud KE, Ewing SK, Cawthon PM, Fink HA, Taylor BC, Cauley JA, et al. A Comparison of Frailty Indexes for the Prediction of Falls, Disability, Fractures and Mortality in Older Men. J Am Geriatr Soc. 2009;57(3):492-8. doi:10.1111/ j.1532-5415.2009.02137.x.

22. Beswick AD, Rees K, Dieppe P, Ayis $S$, Gooberman-Hill R, Horwood J, et al. Complex interventions to improve physical function and maintain independent living in elderly people: a systematic review and meta-analysis. Lancet. 2008;371(9614):725-35. doi: $10.1016 / S 0140-6736(08) 60342-6$.

23. Ping-Hsueh Lee, Yow-Shan Lee, DingCheng Chan. Interventions targeting geriatric frailty: A systemic review. J Clin Gerontol \& Geriatr. 2012;3(2):4752. doi:10.1016/j.jcgg.2012.04.001

24. Yamada M, Arai H, Sonoda T, Aoyama T. Community-based exercise program is cost- effective by preventing care and disability in Japanese frail older adults. J Am Med Dir Assoc. 2012;13(6):50711. doi: 10.1016/j.jamda.2012.04.001.

25. Chou CH, Hwang CL, Wu YT. Effect of exercise on physical function, daily living activities, and quality of life in the frail older adults: a meta-analysis. Arch Phys Med Rehabil. 2012;93(2):237-44. doi: 10.1016/j.apmr.2011.08.042.

26. Cesari M, Vellas B, Hsu FC, Newman $A B$, Doss $H$, King AC, et al. A physical activity intervention to treat the frailty syndrome in older persons-results from the LIFE-P study. J Gerontol A Biol Sci Med Sci. 2015;70(2):216-22. doi: 10.1093 /gerona/glu099.

27. Kim CO, Lee KR. Preventive effect of protein-energy supplementation on the functional decline of frail older adults with low socioeconomic status: a community-based randomized controlled study. J Gerontol A Biol Sci Med Sci. 2013;68(3):309-16. doi: $10.1093 /$ gerona/gls167.
28. Tieland M, van de Rest O, Dirks ML, van der Zwaluw N, Mensink M, van Loon LJ, de Groot LC. Protein supplementation improves physical performance in frail elderly people: a randomized, double-blind, placebocontrolled trial. J Am Med Dir Assoc. 2012 ;13(8):720-6. doi: 10.1016/j. jamda.2012.07.005

29. Tieland M, Dirks ML, van der Zwaluw N, Verdijk LB, van de Rest O, de Groot LC, et al. Protein supplementation increases muscle mass gain during prolonged resistance-type exercise training in frail elderly people: a randomized, double-blind, placebocontrolled trial. J Am Med Dir Assoc. 2012;13(8):713-9. doi: 10.1016/j. jamda.2012.05.020.

30. Muir SW, Montero-Odasso M. Effect of vitamin D supplementation on muscle strength, gait and balance in older adults: A systematic review and meta-analysis. J Am Geriatr Soc. 2011;59(12):2291-300. doi 10.1111/j.1532-5415.2011.03733.x.

31. Daniels R, van Rossum E, de Witte L, Kempen GI, van den Heuvel W. Interventions to prevent disability in frail community-dwelling elderly: a systematic review. BMC Health Serv Res. 2008;8:278. doi: 10.1186/14726963-8-278.

32. Vidal EI. Fragilidad en ancianos: perspectivas para la investigación y la práctica en salud pública. Cad Saude Publica. 2014;30(6):1133-5.

33. Vidal EI, Mayoral VF, Villas Boas PJ, Jacinto AF, Fukushima FB. Physical frailty as a clinical marker of biological age and aging. J Am Geriatr Soc. 2015;63(4): 837-8. doi: 10.1111/ jgs. 13356.

34. Finn M, Green P. The Influence of Frailty on Outcomes in Cardiovascular Disease. Rev Esp Cardiol. 2015;68(8):653-6. doi: 10.1016/j. rec.2015.04.005.
35. Iqbal J, Denvir M, Gunn J. Frailty assessment in elderly people. Lancet. 2013;381(9882):1985-6. doi: 10.1016/S0140-6736(13)61203-9.

36. Afilalo J, Alexander KP, Mack MJ, Maurer MS, Green P, Allen LA, et al. Frailty assessment in the cardiovascular care of older adults. J Am Coll Cardiol. 2014;63(8):747-62. doi: 10.1016/j. jacc.2013.09.070.

37. Ekerstad N, Swahn E, Janzon M, Alfredsson J, Lofmark R, Lindenberger $\mathrm{M}$, et al. Frailty is independently associated with short-term outcomes for elderly patients with non-ST-segment elevation myocardial infarction. Circulation 2011;124(22):2397-404. doi: 10.1161/ CIRCULATIONAHA.111.025452.

38. Puls M, Sobisiak B, Bleckmann A, Jacobshagen C, Danner BC, Hunlich M, et al. Impact of frailty on short- and long-term morbidity and mortality after transcatheter aortic valve implantation: risk assessment by Katz Index of activities of daily living. EuroIntervention. 2014;10(5):609-19. doi: 10.4244/EIJY14M08_03.

39. International Diabetes Federation. IDF Global Guideline for Managing Older People with Type 2 Diabetes. Brussels: IDF; 2013.

40. Kerri M, Clough-Gorr. The epidemiology of cáncer and aging. In: Arash Naeim. Managment of cáncer in the older patient. Piladelphia: Elsevier Saunders; 2012. pp16.

41. Handforth C, Clegg A, Young C, Simpkins S, Seymour MT, Selby PJ, et al. The prevalence and outcomes of frailty in older cancer patients: a systematic review. Ann Oncol. 2015;26(6):1091-101. doi:10.1093/annonc/mdu540.

Correspondencia: Tania Tello Rodriguez. Dirección: Av. Honorio Delgado 430, Urb. Ingenieria, San Martín de Porres

Teléfono: (+511) 3190007

Correoelectrónico:tania.tello.r@upch.pe

Nuestros artículos se encuentran indizados en:

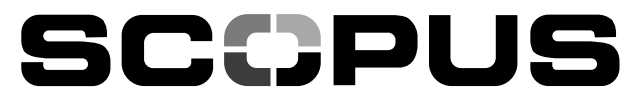

